\title{
Utilización de la simulación clínica en un Máster Interuniversitario en Estudio y Tratamiento del Dolor
}

\author{
E. Roscales ${ }^{1}$, J. M. Maestre ${ }^{2}$, R. Santillán ${ }^{3}$, M. A. Hurlé ${ }^{4}$ e I. del Moral ${ }^{2}$ \\ ${ }^{1}$ Máster Oficial Interuniversitario en el Estudio y Tratamiento del Dolor de la Universidad Rey Juan \\ Carlos y la Universidad de Cantabria. Universidad de Cantabria. Enfermería Área Quirúrgica. Hospital \\ Universitario Valdecilla. Santander. ${ }^{2}$ Servicio de Anestesiología y Reanimación. Hospital Virtual Valdecilla. \\ Santander. ${ }^{3}$ Servicio de Anestesiología y Reanimación. Unidad del Dolor. Hospital Universitario \\ Valdecilla. Santander. ${ }^{4}$ Departamento de Fisiología y Farmacología. Facultad de Medicina. \\ Universidad de Cantabria. Santander
}

Roscales E, Maestre JM, Santillán R, Hurlé MA, del Moral I. Utilización de la simulación clínica en un Máster Interuniversitario en Estudio y Tratamiento del Dolor. Rev Soc Esp Dolor 2014; 21(3): 131-137.

\begin{abstract}
Objectives: To describe our experience integrating simulation based training in an Interuniversitary Master's degree in Pain Study and Management from the University Rey Juan Carlos of Madrid and University of Cantabria, Spain.

Material and methods: After taking the online and on-site modules, students participated in 6 scenarios of simulated clinical immersion, in order to train patient management, clinical diagnosis, and patient safety competencies, in a realistic environment reproducing the actual clinical work.

Results: Five physiotherapists, 1 psychologist, 10 medical doctors, and 9 nurses participated in the simulations during two consecutive editions of the Master. They found the scenarios very realistic and simulation a valuable teaching tool, reporting a global satisfaction of 4.5 over 5 . It was also an opportunity to experiment adverse events of the analgesic that are uncommon to observe during clinical rotations.

Conclusions: Participants were satisfied with the use of clinical simulation as part of the training program of an Interuniversitary Master in Pain Study and Management.
\end{abstract}

Key words: Pain. Education. Simulation. Training. Master.

Recibido: 01-05-13.

Aceptado: 15-09-13.

\section{RESUMEN}

Objetivos: En los sistemas de formación tradicional el entrenamiento se realiza practicando directamente sobre los pacientes durante las rotaciones clínicas. Esta organización tiene como consecuencia que los contenidos docentes dependan de los diferentes lugares de rotación, el tiempo disponible para cada uno de ellos y de la casualidad de encontrar determinadas patologías. Además, la variabilidad de los clínicos puede dificultar la estandarización de contenidos. Nuestro objetivo es describir la experiencia con la integración de la simulación con otros métodos de formación en el marco del Máster Oficial Interuniversitario para el Estudio y Tratamiento del Dolor de la Universidad Rey Juan Carlos de Madrid y la Universidad de Cantabria, con enfoque interprofesional.

Material y métodos: Se integró el aprendizaje online y las sesiones presenciales, con una práctica de 7 horas utilizando la simulación clínica. Se realizaron 4 escenarios con 6 casos clínicos con los objetivos de reconocer y comprender las principales entidades patológicas que cursan con dolor en la práctica clínica. Se utilizaron pacientes estandarizados y simuladores de paciente de alta fidelidad, dependiendo del escenario clínico.

Resultados: Los participantes (5 fisioterapeutas, 1 psicólogo, 10 médicos y 9 enfermeras) consideraron la simulación como herramienta docente muy útil. Encontraron los escenarios realistas y útiles para entrenar el enfoque diagnóstico y terapéutico en pacientes con dolor. Además, fue una oportunidad para experimentar los efectos adversos de los analgésicos que son difíciles de observar durante las rotaciones clínicas, y para que, aquellos alumnos sin competencias profesionales para tratar pacientes, pudieran tomar decisiones clínicas en un ambiente realista y sin riesgo.

Conclusiones: La integración de la simulación clínica dentro de un Máster para el Estudio y Tratamiento del Dolor es percibida de modo muy satisfactorio por los participantes. Proporciona oportunidades para integrar y aplicar de modo práctico los conocimientos adquiridos durante el curso, independientemente de la titulación de origen, de un modo que evoca la realidad y es seguro. Además, facilita la formación específica en la comunicación y el trabajo en equipo interprofesional, cuando 
se toman decisiones para diagnosticar y tratar pacientes con dolor. Es una alternativa a las actuaciones directas sobre los pacientes que están limitadas por el riesgo de comprometer su seguridad clínica.

Palabras clave: Dolor. Docencia. Simulación. Entrenamiento. Máster.

\section{INTRODUCCIÓN}

El Máster Oficial Interuniversitario en Estudio y Tratamiento del Dolor de la Universidad Rey Juan Carlos de Madrid y la Universidad de Cantabria surge ante la necesidad creciente de formar profesionales multidisciplinares en el campo del dolor. Ello es debido a varios factores. Por un lado, el dolor sigue siendo la primera razón por la cual los pacientes acuden a centros de salud y hospitales, y su tratamiento constituye la primera obligación del clínico. Por otro lado, su abordaje a menudo requiere un tratamiento complejo y, en ocasiones, resulta necesaria una aproximación multidisciplinar que incluye médicos de diferentes especialidades, diplomados en enfermería, fisioterapeutas y psicólogos. Asimismo, de acuerdo con el Estudio "Calidad de vida del paciente con Dolor", coordinado por la Sociedad Española del Dolor, el inadecuado tratamiento del dolor en España conlleva un gasto anual de la sanidad pública de 4.500 millones de euros (1).

Dada la gran cantidad de tiempo requerida para completar los estudios de un Máster y, en un intento de facilitar su realización mientas se desarrolla otra labor profesional, existe actualmente una tendencia a ofrecerlos con un formato mixto a distancia y presencial (2). Las plataformas online han experimentado un gran auge en los últimos años y permiten un seguimiento del alumno prácticamente en tiempo real. Sin embargo, el alumno debe complementar la adquisición de conocimientos con prácticas presenciales, que le permitan aplicar y analizar lo aprendido durante las sesiones virtuales (3).

En este Máster, los participantes aprenden las habilidades clínicas necesarias para el manejo del dolor asistiendo a unidades de dolor de hospitales colaboradores en rotaciones de 6 y 24 créditos ECTS. Las rotaciones se combinan con prácticas de laboratorio y seminarios interactivos.

Con los sistemas de formación tradicional, el entrenamiento se realiza practicando directamente sobre los pacientes durante las rotaciones clínicas. Esta organización tiene como consecuencia que los contenidos docentes dependan de los diferentes lugares de rotación, el tiempo disponible para cada uno de ellos y de la casualidad de encontrar determinadas patologías. Además, la variabilidad de los clínicos puede dificultar la estandarización de contenidos (4).
Por otra parte, las actividades que pueden desarrollar los alumnos están condicionadas por la capacitación profesional propia de la titulación de origen, lo que limita la posibilidad de tratar directamente con pacientes.

En este entorno, la simulación clínica surge como una herramienta docente que se complementa con los métodos tradicionales y ayuda a dar solución a algunos de los retos planteados. Por un lado, permite la práctica estandarizada de competencias en un entorno realista y sin comprometer la seguridad del paciente, independientemente de la titulación del participante. Además, se fundamenta en el modo de aprendizaje del adulto, basado en la práctica y la reflexión y permite la práctica repetitiva, adaptándose a las necesidades de aprendizaje de cada alumno en particular (5).

Sin embargo, existen escasas referencias en la literatura en el uso de la simulación como herramienta docente para entrenar el abordaje de pacientes con dolor (6). Y no conocemos másteres en los que esté integrada esta estrategia docente.

El objetivo de este trabajo es describir nuestra experiencia con la integración de la simulación con otros métodos de formación en el Máster Oficial Interuniversitario para el Estudio y Tratamiento del Dolor de la Universidad Rey Juan Carlos de Madrid y la Universidad de Cantabria, con enfoque interprofesional.

\section{MATERIAL Y MÉTODOS}

\section{Descripción del Máster}

El Máster para Estudio y Tratamiento del Dolor de la Universidad Rey Juan Carlos y Universidad de Cantabria se dirige a profesionales relacionados con las Ciencias de la Salud implicados en el tratamiento de pacientes con dolor (anestesiólogos, odontólogos, médicos de atención primaria y de familia, enfermeras de unidades de dolor, fisioterapeutas, psicólogos, etc.), y a licenciados y diplomados interesados en realizar su tesis doctoral en investigación básica y/o clínica en el campo del dolor. En el curso 20102011 se matricularon 98 nuevos alumnos (13\% extranjeros) con un índice de abandono del 12,24\% (7).

El objetivo general del programa es la formación de profesionales capacitados para trabajar en el campo del dolor en sus diferentes vertientes, dependiendo de la formación de origen. El programa se desarrolla a lo largo de dos años (240 créditos), con un formato mixto online y presencial (7), lo que permite a los profesionales adquirir su formación manteniendo sus trabajos de origen. El programa presencial proporciona la oportunidad de poner en práctica los conocimientos adquiridos durante las sesiones multimedia, contextualizar el aprendizaje y contactar con profesores y otros alumnos. La enseñanza presencial en 
este Máster está integrada por 30 créditos ECTS de rotaciones clínicas en unidades de dolor y 30 créditos ECTS de sesiones prácticas y talleres de experimentación en unidades de investigación en dolor (7). Las prácticas presenciales incluyen una jornada de entrenamiento con simulación clínica en el Hospital Virtual Valdecilla.

\section{Criterios seguidos para la selección de las competencias a entrenar mediante simulación clínica}

Se constituyó un equipo de trabajo de 7 profesionales: 2 profesores del Máster (1 farmacóloga, y 1 especialista de anestesiología perteneciente a la Unidad del Dolor), 1 especialista en anestesiología y reanimación e instructora en simulación, 2 enfermeras instructoras en simulación clínica, el director docente y el director del Hospital Virtual Valdecilla. La tarea de este equipo fue: a) identificar dentro del programa teórico del Máster aquellas competencias susceptibles de complementarse y beneficiarse a través de un aprendizaje basado en la propia experiencia en ambientes simulados; b) definir los objetivos docentes de cada actividad; c) diseñar los escenarios; y d) planificar los análisis. El equipo de trabajo seleccionó mediante consenso aquellas actividades que pudiesen añadir mayor valor al programa de formación, siguiendo los criterios que se recogen en la tabla I, que han sido descritos previamente para la selección de competencias susceptibles de entrenamiento mediante simulación clínica (5).

\section{Casos simulados, formato y criterios formativos}

Las actividades online y presenciales se complementaron con una sesión práctica de 7 horas utilizando la simulación clínica, en la que los alumnos participaron en cuatro escenarios en los que se representaron seis casos clínicos.

1. El primer escenario fue diseñado con los objetivos de llevar a cabo una valoración clínica del dolor, reconociendo y evaluando los distintos tipos de dolor en la práctica clínica (nociceptivo, neuropático y mixto), y practicar distintas combinaciones terapéuticas. Se utilizaron dos pacientes estandarizados (actores) que acudían a una consulta ambulatoria con un esguince de tobillo, una neuralgia postherpética y un maniquí que simulaba un paciente con cáncer pulmonar avanzado.

2. El segundo escenario fue diseñado para comprender el enfoque multimodal del tratamiento del dolor, discutir el papel de la analgesia preventiva, y repasar los fundamentos de acción, indicaciones y eficacia de las intervenciones terapéuticas en dolor. Los participantes tuvieron que enfrentarse en la Sala de Recuperación Postanestésica a un paciente
TABLA I. CRITERIOS PARA LA SELECCIÓN DE COMPETENCIAS SUSCEPTIBLES DE ENTRENAMIENTO MEDIANTE SIMULACIÓN CLÍNICA

1. Posibilidad de ser evocadas o replicadas en un entorno simulado

2. Relevancia clínica

3. Posibilidad de evaluación formativa

4. Posibilidad de reducir el tiempo para exponer a los futuros especialistas a la situación clínica

5. Inexistencia de otro método docente ya validado para un entrenamiento eficaz

6. Implique factores dependientes del modo en que está organizado el trabajo

7. Se desarrolle en el seno de un equipo de trabajo

8. Su práctica comprometa la seguridad del paciente

9. Elevada complejidad que haga difícil el aprendizaje en un entorno clínico real

estandarizado con dolor después de ser sometido a una laminectomía, con el objetivo de hacer una valoración tras una operación quirúrgica, y enfocar el tratamiento dependiendo del tipo de anestesia y analgésicos recibidos en el quirófano.

3. En el tercer escenario tuvo el objetivo de integrar en el abordaje del tratamiento del dolor desde la perspectiva de varias especialidades, utilizando además varias alternativas terapéuticas a los métodos tradicionales. Transcurrió en una planta de hospitalización con un paciente estandarizado al que se le había pautado un tratamiento analgésico mediante bomba de perfusión continua después de una resección anterior de recto, para que pudiesen aplicar sus conocimientos a la práctica diaria con un abordaje interprofesional, aprendiendo con otros miembros del equipo, siempre respetando las competencias profesionales propias de cada disciplina.

4. Durante el $4 .^{\circ}$ escenario se experimentaron los efectos adversos de los fármacos analgésicos en quirófano durante una depresión respiratoria causada por sobredosificación de analgésicos y sedantes, en un paciente que estaba siendo sometido a una hernioplastia mediante anestesia espinal. Se utilizaron maniquíes alta fidelidad ya que fue necesario realizar maniobras invasivas para el manejo de la vía aérea (8).

Cada una de las simulaciones clínicas fue gestionada por tres alumnos, mientras el resto de participantes observaba en otra sala a través de un circuito cerrado de televisión. Al finalizar el desarrollo de cada escenario, cuya duración media fue de 20 minutos, se analizaron las actuaciones con todos los participantes en sesiones de 45 minutos. Con el propósito de realizar una evaluación formativa, 
Rev. Soc. Esp. del Dolor, Vol. 21, N. ${ }^{\circ}$ 3, Mayo-Junio 2014

y para analizar las diferencias entre el rendimiento observado y el esperado para cada caso, se utilizó la técnica de análisis y reflexión descrita por el Center for Medical Simulation, Boston (9).

Todos los casos fueron recreados en el Hospital Virtual Valdecilla, una institución docente del Gobierno de Cantabria, cuya misión es mejorar la seguridad del paciente mediante la formación y el desarrollo competencial de los profesionales sanitarios, el desarrollo de investigaciones innovadoras, la promoción del trabajo en equipos interdis- ciplinares y multiprofesionales, a través de la simulación clínica en todas sus aplicaciones (10).

\section{Encuestas para los participantes}

Después del curso se realizó una encuesta anónima de satisfacción a los 25 alumnos, diferenciándose el grupo profesional, con 18 preguntas para contestar según escala de Likert con 5 niveles de respuesta (Tabla II) y 6 preguntas abiertas (Tabla III).

TABLA II. ENCUESTA REALIZADA TRAS EL ENTRENAMIENTO CON SIMULACIÓN

\begin{tabular}{|c|c|c|c|c|c|c|}
\hline \multirow{2}{*}{ Preguntas } & \multicolumn{5}{|c|}{$\begin{array}{l}\text { Número de respuestas } \\
\text { según la escala de Likert }\end{array}$} & \multirow{2}{*}{$\begin{array}{c}\text { Puntuación } \\
\text { media } \pm \\
\text { desviación } \\
\text { estándar }\end{array}$} \\
\hline & 1 & 2 & 3 & 4 & 5 & \\
\hline $\begin{array}{l}\text { 1. Te parecen útiles los ejemplos relacionados con el dolor para tu futuro } \\
\text { profesional }\end{array}$ & 0 & 0 & 2 & 14 & 9 & $4,28 \pm 0,61$ \\
\hline $\begin{array}{l}\text { 2. Mi conocimiento sobre las actuaciones ante diferentes casos de dolor } \\
\text { ha aumentado gracias a la simulación clínica }\end{array}$ & 0 & 0 & 0 & 12 & 13 & $4,52 \pm 0,51$ \\
\hline $\begin{array}{l}\text { 3. La simulación clínica me ha ayudado a saber utilizar los diferentes } \\
\text { métodos de evaluación del dolor en un ámbito real }\end{array}$ & 0 & 0 & 0 & 1 & 24 & $4,96 \pm 0,2$ \\
\hline $\begin{array}{l}\text { 4. La simulación clínica me ha ayudado a saber diferenciar los tipos de } \\
\text { dolor }\end{array}$ & 0 & 0 & 2 & 20 & 3 & $4,04 \pm 0,45$ \\
\hline $\begin{array}{l}\text { 5. La simulación clínica me ha ayudado a completar mi formación sobre } \\
\text { tratamientos específicos de dolor }\end{array}$ & 0 & 0 & 0 & 23 & 2 & $4,08 \pm 0,28$ \\
\hline 6. La experiencia ha mejorado mis habilidades & 0 & 0 & 0 & 16 & 9 & $4,36 \pm 0,49$ \\
\hline $\begin{array}{l}\text { 7. La simulación ha aumentado la confianza en mis habilidades técnicas } \\
\text { ante complicaciones secundarias al tratamiento del dolor }\end{array}$ & 0 & 0 & 7 & 7 & 11 & $4,16 \pm 0,85$ \\
\hline $\begin{array}{l}\text { 8. Ayuda a desarrollar razonamiento crítico y la toma de decisiones ante } \\
\text { diferentes situaciones en un paciente con dolor }\end{array}$ & 0 & 0 & 5 & 17 & 3 & $3,92 \pm 0,57$ \\
\hline 9. El caso se adapta a los conocimientos teóricos que poseo & 0 & 0 & 0 & 5 & 20 & $4,8 \pm 0,41$ \\
\hline 10. La simulación me ha ayudado a integrar teoría y práctica & 0 & 0 & 0 & 6 & 19 & $4,76 \pm 0,44$ \\
\hline 11. Los casos recreaban situaciones reales & 0 & 0 & 0 & 3 & 22 & $4,88 \pm 0,33$ \\
\hline 12. Los escenarios se asemejan a la realidad & 0 & 0 & 0 & 4 & 21 & $4,84 \pm 0,37$ \\
\hline 13. Promueve la participación de todos los alumnos & 0 & 0 & 0 & 2 & 23 & $4,9 \pm 0,28$ \\
\hline 14. Promueve la participación de un grupo multidisciplinar & 0 & 0 & 0 & 1 & 24 & $4,96 \pm 0,2$ \\
\hline $\begin{array}{l}\text { 15. Ayuda a entrenar la toma de decisiones relacionadas con el dolor } \\
\text { dentro de un grupo multidisciplinar }\end{array}$ & 0 & 0 & 0 & 2 & 23 & $4,92 \pm 0,28$ \\
\hline $\begin{array}{l}\text { 16. La simulación clínica afianza conceptos teóricos reforzándoles y/o } \\
\text { poniéndoles en práctica }\end{array}$ & 0 & 0 & 0 & 0 & 25 & $5 \pm 0$ \\
\hline $\begin{array}{l}\text { 17. La experiencia con el simulador aumenta mi seguridad a la hora de } \\
\text { trabajar }\end{array}$ & 0 & 0 & 0 & 4 & 21 & $4,84 \pm 0,37$ \\
\hline $\begin{array}{l}\text { 18. La simulación clínica ha aumentado mi interés por el estudio y } \\
\text { tratamiento del dolor }\end{array}$ & 0 & 0 & 0 & 0 & 25 & $5 \pm 0$ \\
\hline
\end{tabular}


TABLA III. PREGUNTAS ABIERTAS REALIZADAS A LOS PARTICIPANTES TRAS EL ENTRENAMIENTO CON SIMULACIÓN

1. ¿Recuerdas qué contenidos del Máster has aprendido durante la actividad de simulación?

2. ¿Qué te parece el enfoque interprofesional de los casos clínicos realizados durante la actividad de simulación?

3. ¿Qué conocimientos, habilidades u otras prácticas podrás aplicar en tu entorno de trabajo con motivo de la actividad de simulación que has realizado?

4. ¿Cuál es tu pensamiento respecto a tu participación en la actividad de simulación?

5. ¿Cuál es tu opinión respecto a integrar un módulo de simulación en el Máster del Dolor?

6. ¿Sugerirías alguna actividad o tienes alguna sugerencia para este tipo de simulación?

\section{RESULTADOS}

El estudio se realizó con 25 alumnos (5 fisioterapeutas, 1 psicólogo, 10 médicos y 9 enfermeras) que asistieron a las ediciones llevadas a cabo en los cursos académicos 2010-2011 y 2011-2012. Los resultados de las encuestas realizadas tras el entrenamiento con simulación se reflejan en las tablas II y IV. Los datos muestran que el diseño de la actividad permitió entrenar a profesionales de distintas disciplinas de la salud, independientemente de su titulación de origen, sin poner en riesgo a pacientes ni profesionales. Además, la simulación ayudó a enfocar la forma en la que se toman las decisiones en el manejo del paciente con dolor desde un abordaje multidisciplinar.

El grado de satisfacción global de la actividad fue elevado (4,6 sobre 5). Y es que los participantes percibieron que la metodología evoca o replica los aspectos fundamentales de la realidad y los ejemplos relacionados con el dolor les parecieron útiles para su futuro profesional. Además, el entrenamiento reforzó sus conceptos teóricos poniéndolos en práctica y aumentó su seguridad en el trabajo. Los alumnos también refirieron que la simulación clínica les ayudó a completar la formación sobre tratamientos específicos de dolor, ya que ayudó a desarrollar su razonamiento crítico y la toma de decisiones ante diferentes situaciones en un paciente con dolor.

\section{DISCUSIÓN}

Desde el año 1997 se está utilizando la simulación clínica de alto realismo como herramienta formativa en distintos programas de la especialidad de Anestesiología, Reanimación y Terapéutica del Dolor en nuestro
TABLA IV. RESPUESTAS MÁS COMUNES A LAS PREGUNTAS ABIERTAS TRAS LAS SESIONES DE SIMULACIÓN

- Se asemeja a la vida real

- Ayuda a trabajar en equipo

- Experiencia positiva

- Una excelente forma de afianzar conceptos

- Lo pondría una semana entera no sólo un día

- Es básico en un Máster de estas características

- Un ejercicio práctico que posteriormente ayuda a la praxis diaria

- Es completo ya que sirve para cada ámbito práctico del dolor

- Me ha permitido saber cómo actuar en diferentes situaciones

país (11). Desde el año 2005 se emplea de modo integrado y estructurado en el programa docente de los residentes de anestesiología y reanimación. Desde el punto de vista del tratamiento del dolor, se han diseñado actividades para entrenar la toma de decisiones en analgosedación en el paciente crítico $(6,12)$, y para entrenar el manejo de la analgesia perioperatoria en programas de residentes (5). Sin embargo, no hemos encontrado referencias en la literatura que describan su empleo en másteres de postgrado dirigidos al manejo del dolor.

En relación al proceso de diseño y creación de los escenarios, este comienza con el estudio de las necesidades de entrenamiento y la determinación de los objetivos. En nuestra experiencia este es el elemento clave para estructurar y comprender el proceso de aprendizaje. A la hora de elegir el simulador en nuestro caso establecimos que el currículum guiase las características del escenario y la tecnología a emplear, y no al revés. Se decidió construir escenarios contextualizados donde las situaciones clínicas se plantean con una comprensión del entorno social, ambiental y organizativo que rodea al paciente, todo ello con el objetivo de que se puedan entrenar habilidades de pensamiento superiores como la conceptualización, reflexión o pensamiento crítico. También ello posibilita entrenar la interacción con los miembros del equipo y con las condiciones del sistema organizativo (8).

Los participantes en esta experiencia consideraron la simulación como herramienta docente muy útil dentro del Máster en Estudio y Tratamiento del Dolor porque facilita la aplicación, análisis crítico y evaluación de los conocimientos adquiridos durante los módulos teóricos. Debido a la presión asistencial y la falta de tiempo para la reflexión sobre las actuaciones realizadas, resulta un reto acometer la formación de los participantes en el entorno clínico durante sus rotaciones y, en este sentido, la simulación proporcionó un complemento válido (13). Por otra parte, permitió experimentar con casos clínicos concretos, que durante las rota- 
ciones clínicas son difíciles de observar (como por ejemplo los efectos adversos de los analgésicos) y manejar casos complejos que de modo habitual sólo son tratados por especialistas con experiencia. También ofrece la posibilidad de mejorar la comunicación, entrenar planificación de estrategias y la integración de los profesionales para trabajar en equipo de una manera eficiente $(14,15)$.

El Máster no tiene como objetivo proporcionar capacitaciones profesionales diferentes de las que proporciona la titulación de origen. Sin embargo, esta metodología supuso una oportunidad para los profesionales no implicados en actividades clínicas de tratar con pacientes y para todos de poder tomar decisiones clínicas con un abordaje multidisciplinar de modo estandarizado en un entorno realista y seguro, en el que se pueden cometer errores sin riesgo (16).

Estos resultados coinciden con los mostrados por otros autores al entrenar otros tipos de competencias clínicas. Es destacable que lo aprendido en el entrenamiento se transfiere al entorno de trabajo (17) y se retiene en el tiempo por periodos más largos que cuando se utilizan métodos tradicionales (18). Además, existe una evidencia creciente de que los equipos entrenados mediante simulación clínica tienen menos complicaciones cuando se comparan a los formados por métodos tradicionales. Así, por ejemplo, en la atención al parto un mejor Apgar al nacer los bebés y una menor incidencia de parálisis cerebral, o en la inserción de catéteres venosos centrales una disminución de bacteriemia asociada a catéter $(19,20)$.

Los alumnos destacaron que este nuevo método docente les ayudó a completar su formación y aumentar su seguridad a la hora de trabajar. Ello es consistente con el hecho de que esta metodología, basada en la experiencia personal seguida de la reflexión, se adapta al modo de aprendizaje del adulto y puede repetirse hasta adaptarse a la curva de aprendizaje de cada persona en particular (21).

Los principales factores limitantes de esta práctica docente estriban en la dificultad de la organización de los equipos y los elevados costes derivados de la formación del personal docente implicado, y de la adquisición y mantenimiento de los equipos de simulación (22).

En conclusión, la integración de la simulación clínica dentro de un Máster para el Estudio y Tratamiento del Dolor es percibida de modo muy satisfactorio por los alumnos. Proporciona oportunidades para integrar y aplicar de modo práctico los conocimientos adquiridos durante el curso, independientemente de la titulación de origen, de un modo realístico y seguro.

\section{CORRESPONDENCIA:}

José M. ${ }^{\mathrm{a}}$ Maestre

Hospital Virtual Valdecilla

Avda. de Valdecilla, s/n

39008 Santander

e-mail: jmmaestre@hvvaldecilla.es

\section{BIBLIOGRAFÍA}

1. Dolor SEd. Manual de Aplicación del EspidiTest para la terapéutica del dolor en España. Madrid: Instituto Zambón; 2005 [1 junio 2013]; Available at: http://www.institutferran. org/documentos/espiditest/espicompleta.pdf.

2. Mills AC. Evaluation of online and on-site options for master's degree and post-master's certificate programs. Nurse Educator 2007;32(2):73-7.

3. Waldrop MM. Online learning: Campus 2.0. Nature 2013; 495(7440):160-3.

4. Maestre JM, Alonso A. Interdisciplinary human simulation. In: Wilson L, Rockstraw L, editors. Human Simulation for Nursing and Health Professions. New York: Springer Publishing Company; 2011.

5. Sancho R, Rabago JL, Maestre JM, Del Moral I, Carceller JM. Integración de la simulación clínica en el programa formativo de la especialidad de Anestesiología y Reanimación. Rev Esp Anestesiol Reanim 2010;57(10):656-63.

6. Brenner GJ, Newmark JL, Raemer D. Curriculum and cases for pain medicine crisis resource management education. Anesthesia and Analgesia 2013;116(1):107-10.

7. Carlos URJ. [cited 201331 mayo 2013]; Available at: http://www.urjc.es/estudios/masteres_universitarios/ciencias_salud/estudio_tratamiento_dolor/.

8. Maestre JM, Sancho R, Rabago JL, Martínez A, Rojo E, Del Moral I. Diseño y desarrollo de escenarios de simulación clínica: análisis de cursos para el entrenamiento de anestesiólogos. Educ Med 2013;16(1):49-57.

9. Rudolph JW, Simon R, Dufresne RL, Raemer DB. There's no such thing as "nonjudgmental" debriefing: A theory and method for debriefing with good judgment. Simulation in healthcare. Journal of the Society for Simulation in Healthcare 2006;1(1):49-55.

10. Hospital virtual Valdecilla. [cited 201331 mayo 2013]; Available at: http://www.hvvaldecilla.es.

11. Del Moral I, RabanaL JM, Díaz de Terán C. Simuladores en Anestesia. Rev Esp Anestesiol Reanim 2001;48:415-22.

12. González AM, Maestre JM, Villanueva MA, Fidalgo I, Rodríguez-Caballero A, Del Moral I. Aprendizaje y manejo de nuevos agentes farmacológicos en medicina. Utilidad de los simuladores a escala real. Rev Esp Anestesiol Reanim 2005;52(Extraordinario):193.

13. Sancho R, Maestre JM, Del Moral I. Manejo de las crisis. Papel de la simulación en la seguridad del paciente. Rev Esp Anestesiol Reanim 2012;59(Supl. 2):S53-S59.

14. Hernández Pinto P, Odriozola Feu JM, Maestre Alonso JM, López Sánchez M, Del Moral Vicente Mazariegos I, De Miguel Sesmero JR. Entrenamiento de equipos interdisciplinares en urgencias obstétricas mediante simulación clínica. Progresos de Obstetricia y Ginecología 2011;54(12):618-624.

15. Willson H. Factors affecting the administration of analgesia to patients following repair of a fractured hip. Journal of Advanced Nursing 2000;31(5):1145-54.

16. Maestre JM, Del Moral I. A view at the practical application of simulation in professional education. Trends in Anaesthesia and Critical Care 2013;3:146-51.

17. Kuduvalli PM, Parker CJR, Leuwer M, Guha A. Retention and transferability of team resource management skills in anaesthetic emergencies: The long-term impact of a highfidelity simulation-based course. European Journal of Anaesthesiology 2009;26(1):17-22.

18. Boet S, Borges BCR, Naik VN, Siu LW, Riem N, Chandra $\mathrm{D}$, et al. Complex procedural skills are retained for a mini- 
mum of $1 \mathrm{yr}$ after a single high-fidelity simulation training session. Br J Anaesth 2011;107(4):533-9.

19. Draycott T, Sibanda T, Owen L, Akande V, Winter C, Reading $\mathrm{S}$, et al. Does training in obstetric emergencies improve neonatal outcome? BJOG: An international Journal of Obstetrics and Gynaecology 2006;113(2):177-82.

20. Parker CS. Simulation and quality improvement in anesthesiology. Anesthesiology Clinics 2011;29(1):13-28.
21. Rudolph JW, Simon R, Raemer DB, Eppich WJ. Debriefing as formative assessment: Closing performance gaps in medical education. Academic emergency medicine. Official Journal of the Society for Academic Emergency Medicine 2008;15(11):1010-6.

22. Rothgeb MK. Creating a nursing simulation laboratory: a literature review. The Journal of Nursing Education 2008;47(11):489-94. 